\title{
Heat (mass) transfer between an impinging jet and a rotating disk
}

\author{
Y.-M. Chen, W.-T. Lee, S.-J. Wu
}

\begin{abstract}
The aim of this experimental study is to investigate the heat (mass) transfer of a rotating disk with an impinging circular jet. To facilitate the experiments, the naphthalene sublimation technique was employed. In order to analyze the results, measurements of the heat (mass) transfer of a stationary disk with an impinging jet and a rotating disk without jet impingement were also made. From the experimental results, it is found that the heat (mass) transfer are precisely divided into three regimes, namely the impingement dominated regime; the mixed regime and the rotation dominated regime. Correlation of Sherwood number of a rotating disk with jet impingement is also proposed in the present work.
\end{abstract}

\section{List of symbols \\ A constant (301.6247), dimensionless \\ $A_{s} \quad A_{1}=791.4937 ; A_{2}=-8.2536 ; A_{3}=0.4043$, di- mensionless \\ C correlation constant, dimensionless \\ $D \quad$ Diameter of circular jet (m) \\ $D_{f} \quad$ Diffusion coefficient of Naphthalene into air $\left(\mathrm{m}^{2} / \mathrm{s}\right)$ \\ $\mathrm{E}_{s}(x) \quad x=(2 T-574) / 117 ; E_{1}(x)=x ; E_{2}(x)=2 x^{2}-1 ;$ $E_{3}(x)=4 x^{3}-3 x$, dimensionless \\ $l_{\mathrm{sb}} \quad$ the rate of change in naphthalene thickness due to sublimation $(\mathrm{m} / \mathrm{s})$ \\ $h$ local heat transfer coefficient $\mathrm{W} /\left(\mathrm{m}^{2} \mathrm{~K}\right)$ \\ $h_{j} \quad$ mass transfer coefficient of a rotating disk caused by jet $(\mathrm{m} / \mathrm{s})$ \\ $h_{r j} \quad$ mass transfer coefficient of rotating disk with jet impingement $(\mathrm{m} / \mathrm{s})$ \\ $h_{r} \quad$ mass transfer coefficient of a disk rotating in still air $(\mathrm{m} / \mathrm{s})$ \\ $h_{m} \quad$ local mass transfer coefficient $(\mathrm{m} / \mathrm{s})$ \\ $H \quad$ jet tube-to-disk spacing (m) \\ $\mathrm{Nu}$ Nusselt number, dimensionless \\ $\mathrm{Nu}_{r} \quad$ pure rotational Nusselt number (hR/k), dimen- sionless \\ $\mathrm{Nu}_{r j}$ jet-Rotational Nusselt number, dimensionless \\ $\operatorname{Pr} \quad$ Prandtl Number $(v / \alpha)$, dimensionless \\ $P_{V} \quad$ the vapor pressure of the Naphthalene at $T_{W}\left(\mathrm{~N} / \mathrm{m}^{2}\right)$ \\ $Q_{j} \quad$ the jet volumetric flow rate $\left(\mathrm{m}^{3}\right)$}

Received on 12 January 1998

Y.-M. Chen, W.-T. Lee, S.-J. Wu

Department of Mechanical Engineering

National Taiwan University, Taipei, Taiwan 106, ROC

Correspondence to: Y.-M. Chen

$Q_{p} \quad$ the rotationally induced pumping volumetric flow rate passing the impingement radius $\left(\mathrm{m}^{3}\right)$

Radius of disk (m)

Impingement radial position $(\mathrm{m})$

Ideal gas constant (joul/mole·K)

Re Reynolds number, dimensionless

$\operatorname{Re}_{r} \quad$ Rotational Reynolds number $\left(\omega R^{2} / v\right)$, dimensionless

$\mathrm{Re}_{j} \quad$ Jet Reynolds number (UD/v), dimensionless

Sc Schmidt number $\left(v / D_{f}\right)$, dimensionless

Sh Sherwood number $\left(h_{\mathrm{m}} R / D_{f}\right)$, dimensionless

$\mathrm{Sh}_{r} \quad$ Pure rotational Sherwood number $\left(h_{\mathrm{r}} R / D_{f}\right)$, dimensionless

$\mathrm{Sh}_{j} \quad$ Pure jet Sherwood number $\left(h_{j} \mathrm{R} / D_{f}\right)$, dimensionless

$\mathrm{Sh}_{r j} \quad$ Jet-Rotational Sherwood number, dimensionless

$T$ Temperature (K)

$T_{W} \quad$ is the surface temperature of the naphthalene $(\mathrm{K})$

$U$ Average exit velocity of the jet $(\mathrm{m} / \mathrm{s})$

\section{Greek symbols}

$\alpha$

$\omega$

$v$

$\rho_{0}$

$\rho_{\mathrm{s}}$

$\rho_{v w}$

Thermal diffusivity $\left(\mathrm{m}^{2} / \mathrm{s}\right)$

Rotational speed of disk ( $\mathrm{rad} / \mathrm{s})$

Kinematic viscosity of air $\left(\mathrm{m}^{2} / \mathrm{s}\right)$

the density of the local naphthalene vapor density in the free stream $\left(\mathrm{kg} / \mathrm{m}^{3}\right)$

the density of solid naphthalene $\left(\mathrm{kg} / \mathrm{m}^{3}\right)$

$\rho_{v w}$ the local naphthalene vapor density on the disk $\left(\mathrm{kg} / \mathrm{m}^{3}\right)$

1

\section{Introduction}

Heat transfer on rotating disks is a commonly occurring topic in convection heat transfer, especially in rotating machinery. Jet impingement is known to be attractive as means of intensifying convective processes. When impinging jets are applied to the rotating disks, they can produce very high local heat or mass transfer rates. The rotating disk induces on its surface an axisymmetric wall jet which interacts with the impinging jet to produce a cross-flow effect, deforming the jet trajectories and consequently deforming the distribution of impingement heat/mass transfer rates. Applications involving impingement cooling of rotating surfaces are found in many cooling processes, such as in bearing and gear cooling and in the cooling of gas turbine disks. In most of these applications, it is important to have knowledge of the disk temperature and the ability to control it. This means also a necessity of accurate and detailed knowledge of the con- 
vection heat transfer between an impinging jet and a rotating disk.

Despite the recognized need for detailed heat transfer information on rotating surfaces, its acquisition has been slowed and is still incomplete because of the expense and complexity involved with making local heat transfer measurements on rotating surfaces. Conventionally such measurements involve mounting heat flux gauges, or spot heaters and thermocouples, on the disk surface, and transmitting electrical power and measurement signals from and to the rotating apparatus through slip rings. The expense and time factors involved usually result in testing of only limited geometric arrangements and flow conditions.

Owen and Rogers [1] provide a concise review of literature addressing both the fluid mechanics and heat transfer aspects of the subject through 1989. There is only sparse local heat transfer information available, which can be found in studies by Popiel et al. [2], Metzger \& Grochsowsky [3], Metzger et al. [4], Bogdan [5], Popiel \& Boguslawski [6], Metzger et al. [7]. By using the transient technique Bogdan [5] made measurements of local heat transfer. In his paper, correlation equations for local Nusselt number in laminar and turbulent regions were proposed. However, in his equations, the effect of the ratio of the radius of impingement to the jet diameter $(R / D$ as shown in Fig. 4 and Fig. 5) was not considered. Moreover, his results were obtained on the nonisothermal surface of the disk; therefore, the uncertainty of his results must be high. The measurements of local heat transfer done by Popiel \& Boguslawski [6] were carried out using a ringshaped h-calorimeter. With the aid of smoke flow visualization, three regimes of the impinging round jet and rotationally induced disk pumping flow interaction have been distinguished: Jet impingement dominated regime; mixed regime; and rotation-dominated regime. In their study, heat transfer correlating equation has been given for the jet impingement dominated regime only. Recently, Brodersen \& Metzger [8], Brodersen et al. [9] [10] concentrated their studies on the fluid flow.

From the above literature review, it is found that proper heat transfer correlation, covering the three flow regions are still lacking. Also, criterion for the flow regime transition proposed in the literature need further elucidation. The objective of the present work has been to fulfil these tasks. In this study, experimental investigation has been carried out by means of the naphthalene sublimation technique. By using this technique, the difficulties and uncertainties that may occur in the conventional heat transfer measuring system can be circumvented. To analyze the results, experiment on a rotating disk without impinging jet and experiment on a stationary disk with jet impingement were also conducted.

\section{2}

\section{Experimental apparatus and procedure}

Figure 1 shows schematically the main parts of the experimental equipment employed in the present study. A test disk with a diameter of $240 \mathrm{~mm}$ was used in the measurements. A sheet of naphthalene with thickness of $5 \mathrm{~mm}$ was coated on the upper surface of the disk, and

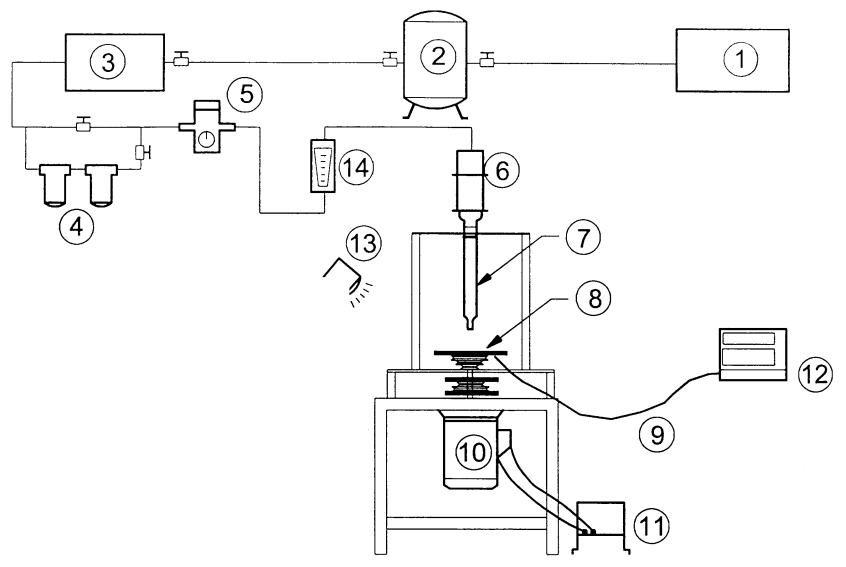

Fig. 1. Schematic of apparatus for the experiment of the plate rotating with the impinging jet. 1 Screw compressor, 2 Air tank 3 Air drier, 4 Filter, 5 pressure regulator, 6 honeycomb and screens, 7 impinging jet, 8 test disk, 9 thermocouple, 10 motor, 11 frequency regulator, 12 recorder, 13 stoboscope, 14 flowmeter

then, the disk was attached to the end of a vertical shaft and driven by a variable-speed electric motor with speed range from 60 to $4000 \mathrm{rpm}$. The rotating speeds were measured by means of a stroboscope. The nozzle, which affixed perpendicularly to the test disk at a desired position, was a circular jet of $7.5 \mathrm{~mm}$ inner diameter and was fitted with a thermocouple to indicate the jet temperature. The aluminum jet nozzle was designed according to the hand-book of ASME (Long Radius Nozzle)[11]. Compressed air, after filtering and drying, was supplied to the rotating disk.

All the measurements ran in a sufficiently large and airconditioned room. By continuously measuring the room temperature with thermocouples, the variation of temperature during each test run had been strictly kept within $1^{\circ} \mathrm{C}$. Before and after the run, naphthalene surface level was measured by use of an automated two-axis data acquisition system. This system was designed and constructed to fulfill the requirements of mass transfer measurements, summarized as precise positioning, accurate surface elevation reading and fast data acquisition. This system consists of a two-axis positioning stand, a depth gauge, a PC and a motor controller system. A schematic diagram of the configuration is shown in Fig. 2. A standard IEEE-488 bus interface was used to transmit data between the components of the system. Since the local mass transfer rate was determined from the difference in naphthalene surface profiles measured before and after exposure to the flow, the test object should be precisely installed on the positioning mechanism. The positioning mechanism consisted of an X-Y table. Stepping motors drove the movement of the two-axis stand. Each motor was equipped with an encoder and was driven by a stepping motor driver. The travel distance in both $\mathrm{X}$ and $\mathrm{Y}$ directions was $130 \mathrm{~mm}$. The X-Y table had positional repeatability over this range of $0.46 \mu \mathrm{m}$. Since the average sublimation depth for the mass transfer measurement was about $200 \mu \mathrm{m}$, a high resolution depth gauge, linear variable differential transformer (LVDT), was used. Its repeatability, according to the manufacturer's specification, 
was $\pm 0.1 \mu \mathrm{m}$ and its measurement range was $0.5 \mathrm{~mm}$. The electronic signal in response to the gauge tip displacement was amplified and converted into a DC voltage that was read then by a digital multimeter.

\section{3}

\section{Data reduction procedure}

The rotational Reynolds number $\left(\mathrm{Re}_{r}\right)$ and jet Reynolds number $\left(\mathrm{Re}_{j}\right)$ are the governing parameters in the present study. They are defined as $\mathrm{Re}_{r}=\omega R^{2} / v$ and $\mathrm{Re}_{j}=U D / v$, where $R$ is the distance between the jet center and the disk center, $\omega$ is the rotating speed of the disk, $D$ is the diameter of the circular jet, and $U$ is the average exit velocity of the jet. The values of $U$ were obtained from the volumetric flow rate measured by a flow meter. A LDA system was also used to check the value of $U$. The experimental parameters used in the present study are listed in Table 1.

In the present study naphthalene sublimation technique was used to measure the mass transfer coefficient instead of the heat transfer coefficient. The value of the heat transfer coefficient was calculated by applying the heat/ mass transfer analogy. The validity of this analogy was discussed in Goldstein \& Karni [12]. The mass transfer coefficient is defined as

$h_{m}=l_{\mathrm{sb}} \rho_{s} /\left(\rho_{v w-} \rho_{0}\right)$

where $h_{m}$ is the local mass transfer coefficient, $l_{\mathrm{sb}}$ is the rate of change in naphthalene thickness due to sublimation, $\rho_{s}$ is the density of solid naphthalene, $\rho_{v w}$ is the local naphthalene vapor density on the disk, and $\rho_{0}$ is the

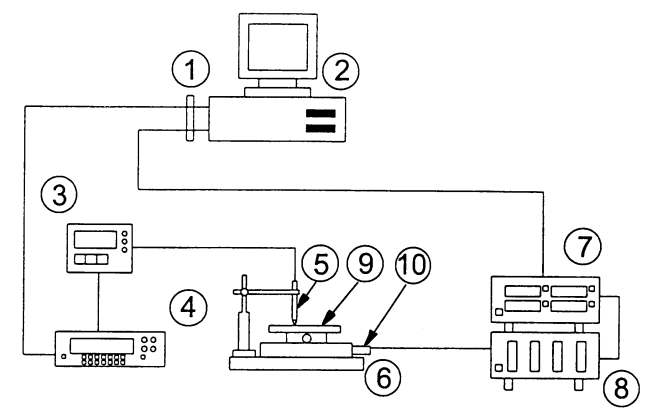

Fig. 2. Automatic data acquisition system. 1 IEEE - 488, 2 IBM PC/XT, 3 LVDT, 4 voltage meter, 5 Probe, 6 stage fixed frame, 7 MC4 stepping motor controller, $8 \mathrm{MC} 4$ controller, 9 measurement plate, 10 stepping motor

Table 1. The list of parameters used in the present study

\begin{tabular}{rrrl}
\hline$\omega(\mathrm{rpm})$ & $\mathrm{Re}_{j}$ & $R / D$ & $H / D$ \\
\hline 500 & 2000 & 0 & 5 \\
1000 & 4000 & 3 & \\
1500 & 6000 & 6 & \\
2000 & 11000 & 9 & \\
2500 & 16500 & 12 & \\
3000 & 25000 & & \\
3500 & 40000 & & \\
4000 & 60000 & & \\
& 80000 & & \\
& 100000 & & \\
\hline
\end{tabular}

density of the local naphthalene vapor density in the free stream (which is equal to zero in the test condition). The naphthalene vapor density on the disk in Eq. (1) can be obtained by applying the ideal gas law

$P_{v}=\rho_{v w} R T_{w}$

The equation that proposed by Ambrose et al. [13] is used to determine the vapor pressure of naphthalene, $P_{v}$.

$T \log P_{v}=0.5 A+\sum_{s=1}^{3} A_{s} E_{s}(x)$

This equation has been widely used in other mass transfer measurement, like Souza [14], Goldstein \& Cho [15]. The mass transfer coefficient can be nondimensionalized by the following equation:

$\mathrm{Sh}=h_{m}\left(D / D_{f}\right)$

where $D_{f}$ is the diffusivity of naphthalene vapor and $D$ is the nozzle diameter. Sherwood number can be transformed to Nusselt number $(\mathrm{Nu})$ for heat transfer by using the analogy between the two processes as

$\mathrm{Nu}=\operatorname{Sh}(\operatorname{Pr} / \mathrm{Sc})^{n}$

At $T=298.15 \mathrm{~K}$, the value of Schmidt number is 2.28 as suggested by Chen et al. [6], and the value of Prandtl number is 0.7 . The naphthalene sublimation technique makes use of the heat and mass transfer analogy to determine heat transfer coefficients in convection flow by measuring mass transfer coefficients. This is discussed in detail in Souza [14] and Cho et al. [15].

The $\mathrm{Re}_{r}$ and $\mathrm{Re}_{j}$ thus obtained were estimated to have uncertainly less than $2.24 \%$ and $2.08 \%$. The maximum uncertainty of the Sherwood number is estimated to be less then $5.02 \%$ for rotating Reynolds number greater than 1000 according to the method suggested by Kline \& McClintock [17].

\section{4}

\section{Results and discussion}

\section{1}

\section{Heat/mass transfer of a disk rotating in still air}

As a jet impinging on a disk, the dominant heat/mass transfer mechanisms conducted by the impinging jet or the rotating disk should be made out. Hence, the behavior of heat/mass transfer with a jet striking on a stationary disk and a disk rotating in still air should be investigated separately. The experimental work with a jet impinging on a stationary disk was also carried out in the present study. Because of the space limitation of the paper, the results are not presented here.

Figure 3 is a plot of Sherwood number as a function of rotational Reynolds number while the test disk rotating in still air. Obviously, values of the Sherwood number against the rotational Reynolds number indicate the existence of three regions: laminar, transition and turbulent. Correlation between Sherwood number and rotational Reynolds number in the three regions, is made form the present experimental results. The correlation equations can be expressed as: 


$$
\mathrm{Sh}_{r}=0.59 \mathrm{Re}_{r}^{0.5} \quad \mathrm{Re}_{r} \leq 2.0 \times 10^{5} \text { in laminar region }
$$

$$
\begin{gathered}
\mathrm{Sh}_{r}=2 \times 10^{-19} \mathrm{Re}_{r}^{4} \quad 2.0 \times 10^{5} \leq \mathrm{Re}_{r} \leq 2.5 \times 10^{5} \\
\text { in transition region } \\
\mathrm{Sh}_{r}=0.0512 \mathrm{Re}_{r}^{0.8 \quad} \quad \mathrm{Re}_{r} \geq 2.5 \times 10^{5} \\
\text { in turbulent region }
\end{gathered}
$$

The three distinct regions were also given by Bogdan [5] and Popiel et al. [2] from the values of Nusselt number as a function of rotational Reynolds number. The critical rotational Reynolds number to divide these three regions in the present study are close to that reported by Bodgan [5] and Popiel et al. [2].

Results of mass transfer obtained from Kreith et al. [18] and Sparrow \& Chaboki [19] in laminar region are also displayed in Figure 3 for comparison. The correlation equation attained from Kreith et al. [18] has the form, $\mathrm{Sh}_{r}=0.6 \mathrm{Re}_{r}^{0.5}$, and the equation obtained by Sparrow \& Chaboki [19] is expressed as $\mathrm{Sh}_{r}=0.625 \mathrm{Re}_{r}^{0.5}$. Comparing the equations in the literature with the present experimental data, the maximum deviation is $5.9 \%$. The present results show good agreement to that reported by Kreith et al. [18] and Sparrow \& Chaboki [19]. As also can be seen in Fig. 3, the Sherwood number obtained in the present study is in proportion to fourth power of rotational Reynolds number in transition region, and is in proportion to 0.8 th power of rotational Reynolds number in turbulent region. Similar proportion between Nusselt number and rotational Reynolds number in these two regions were also reported by Bogdan [5], Metzger et al. [4] and Popiel et al. [2].

\section{2}

Heat/mass transfer of a rotating disk with jet impingement Figures 4 and 5 show the measured Sherwood numbers as a function of $R / D$ and $\operatorname{Re}_{r}$ with a jet striking on the rotating test disk. The distributions of Sherwood num-

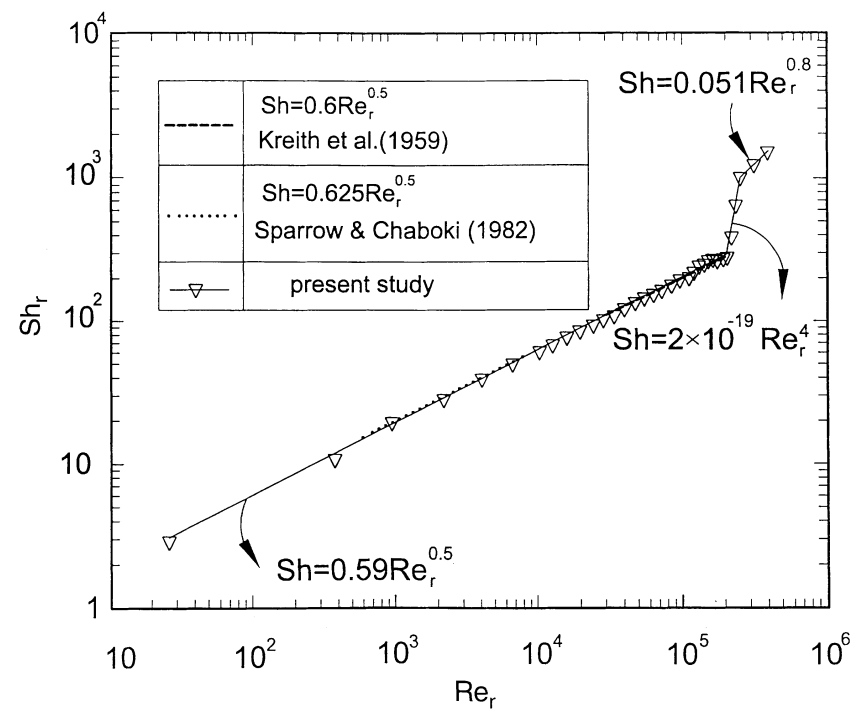

Fig. 3. Local heat/mass transfer of a rotating disk in still air ber at different values of $R / D$ are shown in Figure 4. In this case, $\mathrm{Re}_{j}$ is fixed at 60000 . Obviously, as compared with the result without jet impingement, there is a sudden increase in Sherwood number as $\mathrm{Re}_{r} \leq 2.0 \times 10^{5}$ (the laminar region discussed previously) for the presence of an impinging jet. In the region as $\mathrm{Re}_{r} \leq 2.0 \times 10^{5}$, the Sherwood number remains different constants at different values of $R / D$. The result implies that the increase in rotational Reynolds number causes insignificant influence on the Sherwood number. On the contrary, variations of values $R / D$ lead to consequential effect on the Sherwood number. A smaller value of $R / D$ gives rise to a larger value of Sherwood number as $\operatorname{Re}_{r} \leq 2.0 \times 10^{5}$. It is noteworthy that the transition from laminar region to turbulent region as the disk rotating in still air is ambiguous for the presence of jet. From the value of $\mathrm{Sh}_{r j}$ against $\mathrm{Re}_{r}$ shown in Fig. 4, the value of $\mathrm{Sh}_{r j}$ almost matches that of a disk rotating in still air as the rotational Reynolds number is greater than a critical value. The critical value of rotational Reynolds number is slightly different from the various values of $R / D$.

Figure 5 is a plot of Sherwood number with jet impingement against rotational Reynolds number at various jet Reynolds number. In this case, the value of $R / D$ is kept at 6. The variations of $\mathrm{Sh}_{r j}$ against $\mathrm{Re}_{r}$ display much similarity to that shown in Fig. 4. The value of $\mathrm{Sh}_{r j}$ nearly remains constant as $\operatorname{Re}_{r} \leq 2.0 \times 10^{5}$ at different values of $\mathrm{Re}_{j}$. A larger value of $\mathrm{Re}_{j}$ leads to a larger value of $\mathrm{Sh}_{r j}$ as $\mathrm{Re}_{r} \leq 2.0 \times 10^{5}$. As can be seen from Fig. 5, the value of Sherwood number with jet impingement comes close to that of a disk rotating in still air as rotational Reynolds number is greater than a critical value. The result indicates that the impinging jet produces trivial influence on heat/ mass transfer, as rotational Reynolds number is greater than a critical value. Obviously, influence of jet on laminar heat/mass transfer is much greater than in turbulent. Progress in laminar heat/mass transfer may be attributed to introduction of new amount of fresh air into the disk

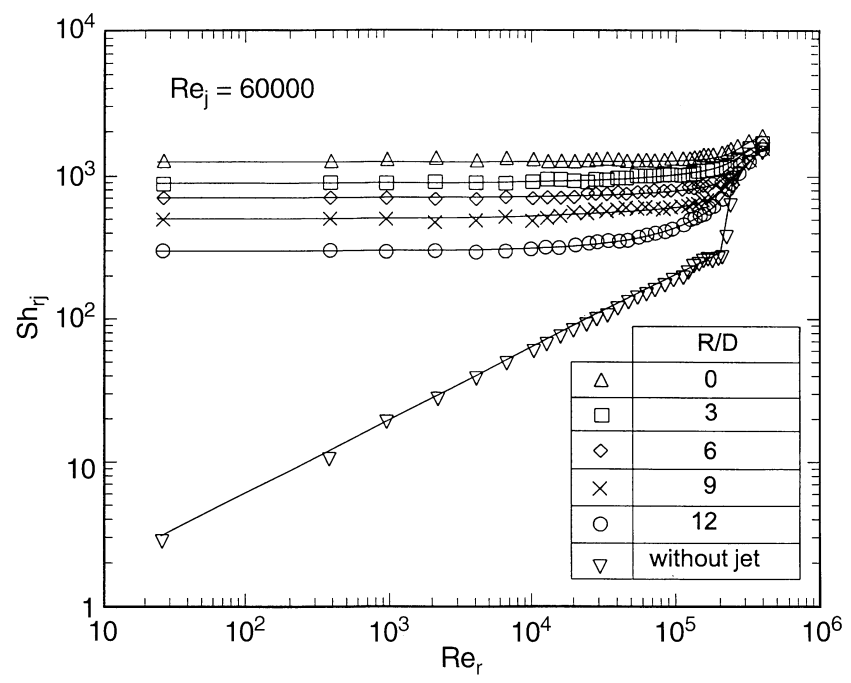

Fig. 4. Local heat/mass transfer of rotating disk with impinging jet at $\mathrm{Re}_{j}=60000$ 


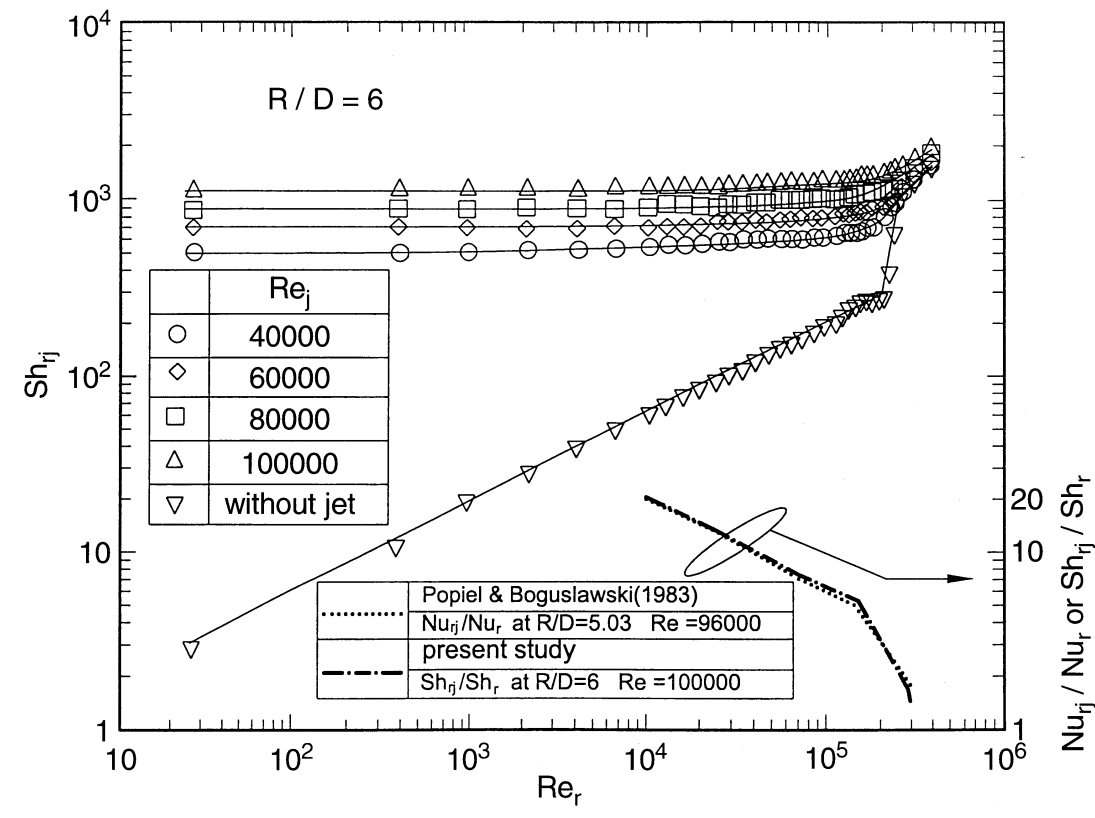

boundary and promotion of turbulent level in the boundary caused by jet.

From the results shown in Figs. 4 and 5, there can exist three regimes of heat/mass transfer. The regime where the Sherwood number remains constant can be called as jet dominated regime since the rotational Reynolds number gives negligible influence on Sherwood number. As rotational Reynolds number is greater than a critical value, the Sherwood numbers measured at various jet Reynolds number come very close to that measured for a disk rotating in still air. The regime can be named as rotation dominated regime since the impingement jet generates incidental influence on heat/mass transfer. A narrow regime, where both rotational disk and impinging jet produce important effect on heat/mass transfer, survives between these two distinct regimes defined previously and can be specified as mixed regime. Precise definition and classification of these three regimes will be made below.

In Fig. 5, the Nusselt number obtained by Popiel \& Boguslawski [6] is also exhibited for comparison. The experimental conditions in Popiel \& Boguslawski [6] are close to that in the present study. From the result shown in Fig. 5, it reveals that the Nusselt number ratio of $\mathrm{Nu}_{r j} / \mathrm{Nu}_{r}$ reported by Popiel \& Boguslawski [6] shows good consistency with the Sherwood number ratio of $\mathrm{Sh}_{r j} / \mathrm{Sh}_{r}$ obtained in the present study.

To make a further discussion of heat/mass transfer of a rotating disk with jet impingement, the following steps deduce correlation of Sherwood number:

1. Calculate $h_{j}=h_{r j}-h_{r}$ at each measurement point, where $h_{\mathrm{j}}$ is the coefficient of mass transfer caused by jet impingement.

2. Find proper correlation equations for $\mathrm{Sh}_{j}$, where $\mathrm{Sh}_{j}$ is defined as $\mathrm{Sh}_{j}=h_{j} R / D_{f}$.

By virtue of the above procedures, the jet Sherwood number is correlated as

$\mathrm{Sh}_{j}=C \operatorname{Re}_{r}^{0.7}(R / D)^{0.1}$

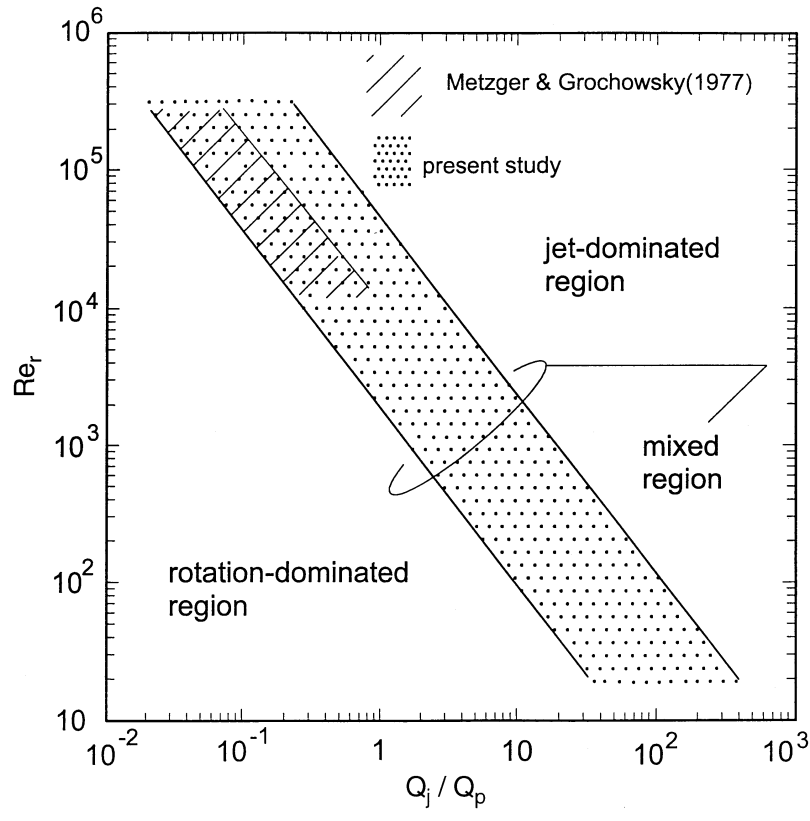

Fig. 6. The correlation of the flow regimes of a rotating disk with impinging jet

It is noteworthy that the Sherwood number of a rotating disk with jet impingement is then expressed as

$\mathrm{Sh}_{r j}=\mathrm{Sh}_{j}+\mathrm{Sh}_{r}$

$$
\begin{aligned}
& =\mathrm{CRe}_{j}^{0.7}(R / D)^{0.1} \quad \text { (jet impinging component) } \\
& + \begin{cases}0.59 \mathrm{Re}_{r}^{0.5}, & \mathrm{Re}_{r}<2.0 \times 10^{5} \\
2 \times 10^{-19} \mathrm{Re}_{r}^{4}, & 2.0 \times 10^{5} \leq \mathrm{Re} \leq 2.5 \times 10^{5} \\
0.051 \mathrm{Re}_{r}^{0.8}, & \mathrm{Re}_{r}>2.5 \times 10^{5}\end{cases} \\
& \text { (rotational component) }
\end{aligned}
$$

From the result shown in Eq.8 (a)(b)(c), the Sherwood number of a rotating disk with jet impingement can be divided in two components. One of the components is 


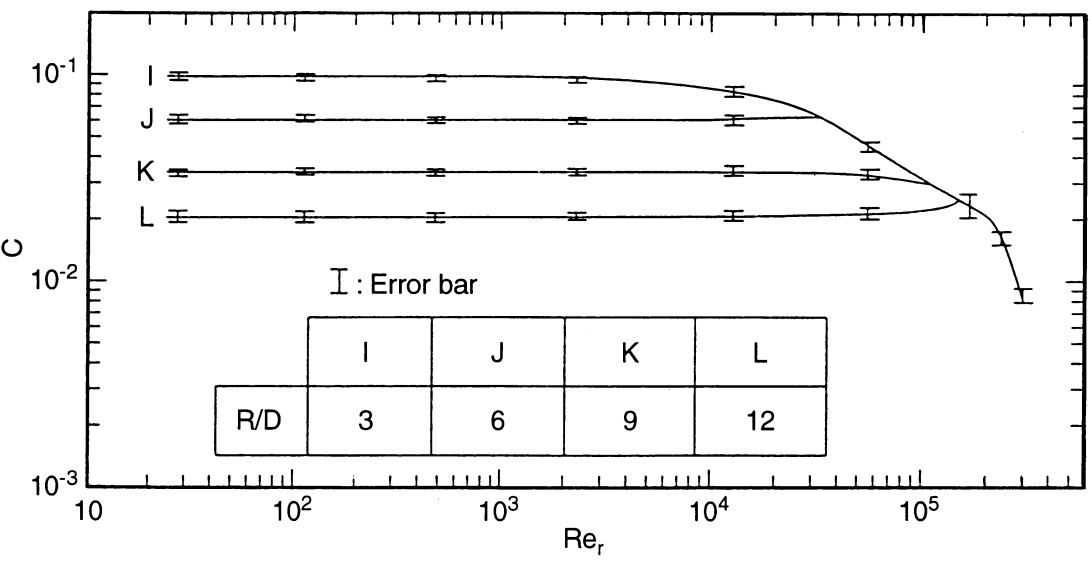

Fig. 7. Correlation of the $\mathrm{C}$ value (Eq. 7) by the impinging jet on the rotating disk guided by the impinging jet, and the other is directed by rotating disk. The value of $\mathrm{C}$ in Eq. (7) is not a constant. Figure 7 shows the values of $C$ as a function of $R / D$ and $\operatorname{Re}_{r}$. The result in Fig. 7 reveals that $C$ is a function of $R / D$ only as $\operatorname{Re}_{r}<10^{4}$; a function of $\operatorname{Re}_{r}$ and $R / D$ as $10^{4} \leq \mathrm{Re}_{r} \leq 1.5 \times 10^{5}$, and a function of $\mathrm{Re}_{r}$ only as $\operatorname{Re}_{r}>1.5 \times 10^{5}$.

\section{3}

\section{Heat/mass transfer regimes of a rotating disk with jet impingement}

The behavior of heat/mass transfer involving a jet impinging on a stationary disk or a disk rotating in still air has been determined. Based on the results discussed previously, flow regimes associated with significant heat/mass transfer mechanisms can be resolved. In order to distinguish the flow regimes, the procedures listed below have been employed:

1. Calculate $Q_{j} / Q_{p}$ at each measured point, where $Q_{j}\left(=\operatorname{Re}_{j} \pi D v / 4\right)$ is the jet volumetric flow rate and $Q_{p}\left(=0.067 \pi r v \mathrm{Re}_{r}\right)$ is the rotationally induced pumping volumetric flow rate passing the impingement radius. The method to calculate $Q_{p}$ was proposed by Schlichting [20].

2. Calculate the value of $h_{r j}-h_{r}$ at each measured point, where $h_{r j}$ is the mass transfer coefficient of a rotating disk with jet impingement and $h_{r}$ is the mass transfer coefficient of a disk rotating in still air.

3. The following criterions are used to distinguish the flow regimes:

$$
\begin{aligned}
& \left(h_{r j}-h_{r}\right) / h_{r j} \leq 10 \% \text { in rotation dominated re- } \\
& \text { gime } \\
& h_{\mathrm{r}} / h_{\mathrm{rj}} \leq 10 \% \text { in jet impingement dominated } \\
& \text { regime } \\
& \left(h_{r j}-h_{r}\right) / h_{r j}>10 \% \text { and } h_{r} / h_{r j}>10 \% \text { in mixed } \\
& \text { regime }
\end{aligned}
$$

Figure 6 shows the correlation attained in terms of the ratio $Q_{j}$ to $Q_{p}$ versus $\operatorname{Re}_{r}$. Each of data point represents conditions at an observed transition point; the resulting band of points separate the rotational and impingement dominated zones. Correlation developed by Metzger \& Grochowsky [3] is also presented in Fig. 7 for comparison. The results reported by Metzger \& Grochowsky [3] were obtained by means of flow visualization experiments. As can be seen in Fig. 6, the left transition line obtained in the present study coincides with that attained from Metzger \& Grochowsky [3], while the right transition line deviation can be attributed to the uncertainty coming from smoke flow visualization and the smaller range of flow conditions in their experiments. The values of $\mathrm{Re}_{r}$ and $Q_{j} / Q_{p}$ pervade much wider ranges than that used in Metzger \& Grochowsky [3]. The ratio of $Q_{j} / Q_{p}$ in the present study varies from $10^{-2}$ to $10^{3}$, while the upper limit of $Q_{j} / Q_{p}$ in Metzger \& Grochowsky [3] is about 1 .

\section{5}

\section{Conclusions}

In the present work, heat/mass transfer of a rotating disk with jet impingement was experimentally examined by virtue of naphthalene sublimation techniques. Distinct divisions of the three heat/mass transfer regimes are made from the experimental results in the present study. Correlation of Sherwood number of a rotating disk with jet impingement is proposed in the present study. It is concluded that the Sherwood number of a rotating disk with jet impingement is the sum of the two components, governed by the impinging jet and the rotating disk respectively.

\section{References}

1. Owen JM; Rogers RH (1989) Flow and Heat transfer in Rotating-Disk Systems. Research Studies Press LTD.1

2. Popiel CO; Tulisica E; Boguslawski L (1974) Heat Transfer From a Rotating Disk in an Impinging Round Air R Jet. Proceedings of the 5th International Heat Transfer Conference 3:212-216

3. Metzger DE; Grochowsky LD (1977) Heat Transfer between an Impinging Jet and a Rotating Disk. ASME. J. of Heat Transfer 99:663-670

4. Metzger DE; Mathis WJ; Grochowsky LD (1979) Jet Cooling at the Rim of a Rotating Disk. ASME. J. of fluid Mech. 101:68-77

5. Bogdan Z (1982) Cooling of a Rotating Disk by Means of an Impinging Jet. Proceedings of the 7th International Heat Transfer Conference, Hemisphere Publishing Corp., Washington, D.C. 3:333-336

6. Popiel CO; Boguslawski L (1986) Local Heat Transfer From a Rotating Disk in an Impinging Round Jet. ASME. J. of Heat Transfer 108:357-364

7. Metzger DE; Bunker RS; Bosch G (1991) Transient Liquid Crystal Measurement of Local Heat Transfer on a Rotating 
Disk with Jet Impingement. ASME. J. of Turbomachinery 113:53-59

8. Brodersen S; Metzger DE (1992) Experimental Investigation of the Flowfield Resulting from the Interaction between an Impinging Jet and a Rotating Disk. Exp. Thermal and Fluid Sci. 5:351-358

9. Brodersen S; Metzger DE; Fernando HJS (1996) Flows Generated by the Impingement of a jet on a Rotating Surface: Part I-Basic Flow Patterns. ASME. J. of Fluid Eng. 118:61-67

10. Brodersen S; Metzger DE; Fernando HJS (1996) Flows Generated by the Impingement of a jet on a Rotating Surface: Part II. Detailed Flow Structure and Analysis. ASME. J. of Fluid Eng. 118:68-73

11. Applied Fluid Dynamics Handbook. ASME. New York, (1959)

12. Goldstein R J; Karni J (1984) The Effect of Wall Boundary Layer on Local Mass Transfer from a Cylinder in Crossflow. ASME. J. of Heat Transfer 106:261-267

13. Ambrose D (1975) The Vapor Pressure of Naphthalene. J. of Chem. Thermodynamics 7:1173-1176

14. Souza MPR (1991) The Naphthalene Sublimation Technique. Exp. Thermal and Fluid Sci. 4:510-523

15. Goldstein RJ; Cho HH (1995) A Review of Mass Transfer Measurements Using Naphthalene Sublimation. Exp. Thermal and Fluid Sci. 4:416-434

16. Chen P H; Miao JM; Jian CS (1996) Technique for Investigating the Temperature Effect on the Diffusion Coefficient of Naphthalene into Air. Rev. Sci. Instrum. (8) 67:2831-2836

17. Kline SJ; McClintock FA (1953) Describing Uncertainties on Single-Sample Experiments. Mechanical Engineering 3-8

18. Kreith F; Taylor JH; Chong JP (1959) Heat and Mass from a Rotating Disk. ASME. J. of Heat Transfer 95-105

19. Sparrow EM; Chaboki A (1982) Heat Transfer Coefficients for a Cup-like Cavity Rotating about Its Own Axis. Int. J. Heat Mass Transfer (9) 25:1334-1341

20. Schlichting H Boundary Layer Theory. Sixth ed., McGraw Hill, New York, 1968 